\title{
Book Review: Handbook of Research on K-12 Online and Blended Learning
}

\author{
Diane Mayse,PhD \\ Blended Learning Data Manager (Nexus Academies) \\ Connections Education
}

Editors: Richard E. Ferdig and Kathryn Kennedy (2014). Handbook of Research on K-12 Online and Blended Learning. Pages: 540. Published by ETC Press. ISBN: 978-1-312-58708-3. The online version is available for no charge at http://press.etc.cmu.edu/content/handbook-research-k-12-online-and-blendedlearning-0. The printed version is $\$ 33.95$.

\section{Introduction}

The Handbook of Research on K-12 Online and Blended Learning, which is edited by Richard E. Ferdig and Kathryn Kennedy, is designed to be a collection of what is currently known about research in the field of K-12 online and blended learning and to serve as a resource for researchers, practitioners, and policy makers.

Richard Ferdig is an accomplished researcher and current professor of Learning and Instructional Technologies at Kent State University. His research, teaching, and service work focus on combining cutting-edge technologies with current pedagogic theory to create innovative learning environments, such as educational games and simulations. Ferdig is also the editor-in-chief of the International Journal of Gaming and Computer Mediated Simulations and the associate editor-in-chief of the Journal of Technology and Teacher Education. He currently serves as a consulting editor for the development editorial board of Educational Technology Research and Development and on the review panel of the British Journal of Educational Technology.

Kathryn Kennedy is an award-winning teacher, researcher, and professor in the area of curriculum and instruction with a focus on educational technology. Kennedy is a senior researcher at the Michigan Virtual Learning Research Institute (MVLRI) — an organization with the goals of supporting new learning models, engaging in active research to inform new policies in online and blended learning, and strengthening the infrastructure for sharing best practices. She was awarded the International Association 
for K-12 Online Learning (iNACOL) Online Learning Innovator Award for Outstanding Research in 2010 and 2012.

The intended audience of this book includes anyone working, researching, teaching, or interested in learning more about K-12 online and blended learning.

The editors' purpose was to create a publicly available, free online resource to begin to synthesize the various strands of research in K-12 online and blended learning into one comprehensive catalogue.

The editors' thesis is that this resource will strengthen further research in the K-12 online and blended learning field by clarifying what is currently known and what is currently unknown; provide empirical resources for researchers, educators, administrators, and policy officials; and begin a process of annually reexamining research in the field.

\section{Organization/Structure}

The Handbook is organized into six sections, each representing a category of K-12 online and blended research: background and history, learner research, content domain, teaching, preparation and mentoring for support roles, and technological innovations.

The first section orients the reader to the historical context of K-12 online and blended learning research as a foundation for understanding the more specific areas of research that follow. The historical overview covers research from the United States and across the world, and reviews policies and research methods used in K-12 online and blended learning.

Each of the remaining topics is presented with an introduction; discussion of the literature; implications for policy, practice, and research; a conclusion; and references. Within each section, two to five authors provide an overview and synthesis of more specific research topics, such as

- learners and learning environments;

- learning in the content domains of math, literature, and physical education;

- teaching, mentoring, and teacher preparation programs;

- administrators, school psychologists, onsite and virtual facilitators, school libraries, and the support role of parents; and

- technological innovations in mobile, open, and personalized learning.

The information Ferdig and Kennedy present is an accurate reflection of the current state of research in each of these domains. The book draws on the research and reflections of key contemporary researchers and provides a solid presentation across several subtopics within each domain, with the goal of creating an ongoing conversation with readers about research in each field. The editors even provide an e-mail address (handbookresearch@gmail.com) so that readers can suggest areas for additional research.

\section{Assessment of Significance to the Field of Distance Education Theory, Research, and/or Practice}

Research presented throughout the book spans from 1994 to the present for internationally based research, and from 2000 to the present for U.S.-based research, with the majority of research cited having been published within the past five years.

By organizing the framework into six broad themes and then selecting a variety of studies representing various subtopics within each theme, the editors give the reader the opportunity to engage with key research ideas across many related areas of interest. 
A major strength is the broad reach of topics combined with a specific focus on exemplar research studies synthesized by the contributors - a collection of the field's leading researchers. In addition, information is presented concisely, and identifies key programs, dates, and relevant details. The book is conveniently organized with descriptive headings and subheadings for easy referencing, and definitions of important terms are provided. Furthermore, each section's introduction connects to the ideas of the sections that came before it, creating smooth transitions throughout.

Another strength of the Handbook is that the editors note additional areas of research they view as missing from each section of the current iteration of the Handbook, which include program evaluation, cultural perspectives and understanding diversity, English language learners, appreciation of change in school cultures, and a continued exploration of the relationships between traditional, online, and blended learning environments. Many relevant reports and research studies are referenced throughout the Handbook in support of its stated collaborative nature.

I hope the editors continue with their mission to expand the areas of research covered in the Handbook and to build upon conversations with readers to determine the future direction and goals of this resource.

As the first work of its kind in this area, directly comparable works do not currently exist. The Evergreen Consulting group publishes the Keeping Pace report each year in partnership with iNACOL. This report provides snapshots of the demographic landscape of online and blended learning and works to continually redefine the various program components of blended and online programs. Nothing currently exists, however, that covers the scope of the Handbook's broader themes, with the goal of centralizing and synthesizing K-12 online and blended learning research.

The Handbook is well designed, well written, and humorous throughout. Ferdig and Kennedy have included research experts from across the field to guide discussions in each category with the reader. The Handbook includes key research resources for each thematic category and suggestions for additional research directions. For these reasons, the Handbook of Research on K-12 Online and Blended Learning is a valuable contribution to the literature of distance education theory, research, and best practices. 